\title{
Preventing Adverse Events of Chemotherapy for Gastrointestinal Cancer by Educating Patients about the Nocebo Effect: A Randomized-controlled Trial
}

Twyla L. Michnevich ( $\triangle$ Twyla.michnevich@tww-berlin.de)

University Medical Center Hamburg-Eppendorf

Yiqi Pan

University Medical Center Hamburg-Eppendorf

Armin Hendi

Asklepios Clinic Barmbek

Karin Oechsle

University Medical Center Hamburg-Eppendorf

Alexander Stein

University Medical Center Hamburg-Eppendorf

Yvonne Nestoriuc

Helmut Schmidt University, University of the Federal Armed Forces Hamburg

\section{Research Article}

Keywords: placebo, nocebo, expectations, chemotherapy, adverse events

Posted Date: October 11th, 2021

DOI: https://doi.org/10.21203/rs.3.rs-926779/v1

License: (c) (i) This work is licensed under a Creative Commons Attribution 4.0 International License. Read Full License 


\section{Abstract}

Background: Adverse events of chemotherapy may be caused by pharmacodynamics or psychological factors such as negative expectations, which constitute nocebo effects. In a randomized controlled trial, we examined whether educating patients about the nocebo effect is efficacious in reducing the intensity of self-reported adverse events.

Methods: $N=49$ and $n=51$ patients (mean age: 60.2 years, $65 \%$ male, $54 \%$ UICC tumour stage IV) with newly-diagnosed gastrointestinal cancer were allocated to a nocebo education and attention control group, respectively.

Results: GLM with adjustments for tumour staging and distress indicated that intensity of adverse events differed at 12-weeks after onset of chemotherapy (mean difference: $4.04,95 \% \mathrm{Cl}[0.72,7.36], p=.02, d=0.48$ ), with lower levels in the nocebo education group. Of these). This was attributable to less non-specific adverse events (mean difference: $0.39,95 \% \mathrm{Cl}[0.04,0.73], p=.03, d=0.44$ ) and a trend towards less specific adverse events in the nocebo education group (mean difference: $0.36,95 \% \mathrm{Cl}[-0.02,0.74], p=.07, d=0.37$ ). We found no difference in adverse events at 10-days follow-up, perceived control of adverse events, or tendency to misattribute non-specific symptoms to the chemotherapy.

Conclusions: This study provides first proof-of-concept evidence for the efficacy of a brief information session in preventing adverse events of chemotherapy. However, results regarding patient-reported outcomes cannot rule out response biases. Informing patients about the nocebo effect may be an innovative and clinically feasible intervention for reducing the burden of adverse events.

Trial registration: retrospectively registered on March 27, 2018 to the German Clinical Trial Register (ID: DRKS00009501).

\section{Background}

The overwhelming majority of patients undergoing chemotherapy for gastrointestinal (GI) cancer is affected by treatment-related adverse events (AEs) $(1,2)$. Aside from impairing patients' quality of life (QoL) $(3,4)$, AEs also decrease treatment adherence (5), and are one of the main reasons for discontinuation (6). Moreover, they significantly add to the costs of cancer treatment (2). Therefore, factors that may contribute to the development and reduction of AEs warrant clinical attention.

Patients' experience of AEs is susceptible to the nocebo effect (7). Here, nocebo effect denotes any adverse response to an active substance that cannot be attributed to its pharmacological effects. In its most tangible form, the nocebo effect occurs after exposure to an inert substance, such as a placebo pill (9). Meta-analysis of clinical cancer trials showed that $10-60 \%$ of patients in the placebo-arms experienced AEs (10), which in fact mirrored those in the active drug arms. Arguably, these AEs are the result of patients' negative expectations, evoked for example during informed consent (IC) (11). The physiological effects of expectations have been underpinned by neurobiological correlates, primarily in nocebo pain modulation $(12,13)$.

Nocebo responses to active drugs more closely resemble clinical routine. Several studies have shown that patients report more AEs when they are informed about potential AEs (14-16). However, many of the AEs from verum drugs are not attributable to pharmacological effects (7). A potential mechanism of this is misattribution of pre-existing or unrelated symptoms (9). In a general population study, the median amount of symptoms (typically day-to-day ailments such as rash or bloating) reported in the past seven days was five, with only $11 \%$ of participants reporting no symptoms [18, see also 19]. These symptoms can be misattributed to new medications (19), especially when patients already experience many symptoms (20). The nocebo effect can also lead to exacerbation of medication-specific AEs. A meta-analysis showed a medium-sized relationship between expecting and experiencing AEs of chemotherapy (21). A further inducer of nocebo resembles the mechanism of classical conditioning: for example, prior exposure to chemotherapy increased the likelihood of pre-treatment nausea $(21,22)$

An innovative means of reducing the nocebo effect suggested by Barsky and colleagues (9) is to inform patients about the nocebo effect. In theory, the awareness that not all symptoms are pharmacological effects of their chemotherapy would allow patients to perceive symptoms as less threatening and therefore more tolerable (9). Specifically, misattribution of non-specific symptoms may be reduced, and perceived control of symptoms as well as treatment expectations improved. Dysfunctional treatment expectations have been shown to influence adverse events of cancer treatment in breast cancer $(23,24)$. In a first study $(25)$, participants with self-reported chronic headache were recruited under the guise of participating in a clinical trial for a headache medication. All participants read a bogus medication leaflet before receiving a placebo pill, and half the patients' leaflets included an explanation of the nocebo effect. Those who received the nocebo effect leaflet reported significantly less AEs than the control group (cf. 26).

In summary, informing about the nocebo effect may be effective in reducing the experience of AEs. This type of intervention is fast, simple, cost-effective and ethically feasible; therefore, it could potentially serve as a component of adverse effect management. Moreover, it requires no alteration to clinical routine or IC. In this study, we therefore examine the efficacy of a nocebo education intervention in a clinical sample

Page 2/17 
receiving verum medication. We hypothesized that patients undergoing chemotherapy for GI cancer would experience less AEs if they were informed about the nocebo effect. This patient population is exposed to considerable distress (27) and at risk for symptom misattribution: on top of possible symptoms from the underlying malignancy, two thirds of patients with GI cancer have comorbidities (28). Suggesting that perceived AEs of chemotherapy are not solely caused by the pharmakon itself may increase patients' perceived self-efficacy in symptom management and reduce misattribution. To examine possible contributing factors to the effect of nocebo education, we assessed patients' perceived control of AEs, their tendency to misattribute symptoms, compliance intention, attitude towards chemotherapy, clinician-rated toxicity and co-medication used to treat AEs. Moreover, we investigated whether optimized treatment expectations mediated hypothesized beneficial effects of nocebo education. As a monitoring information coping style is associated with a higher report of AEs (29), we also assessed the moderating effect of desire for information about AEs.

\section{Methods}

\section{Procedures}

The trial was approved by the University of Hamburg ethics committee (ID: 2015_03) and retrospectively registered on March 27th, 2018 in the German Clinical Trial Register (ID: DRKS00009501). The study was conducted in accordance with the declaration of Helsinki. Enrolment and follow-up assessments took place from 08/2015 to 05/2018 and 10/2015 to 09/2018, respectively.

Oncologists pre-screened patients for eligibility once they were indicated to receive chemotherapy. The study team informed patients verbally and in writing about the overarching goal of the study, namely, to gain insight into expectations about chemotherapy QoL during treatment. All participants gave written informed consent prior to enrolment. The intervention was conducted during or $\leq 24$ hours before first chemotherapy. Patients in the EG received a nocebo education session ${ }^{17}$. In the CG, patients were inquired about the multifaceted aspects of QoL. Both sessions were semi-manualized, conducted by a trained healthcare professional in an empathetic, patient-centred manner, and lasted 20-30 minutes. Details can be found in the protocol ${ }^{17}$.

Concomitant treatments, including psychosocial interventions, radiation therapy and medication, were permitted. Patients in the CG received the nocebo education leaflet by mail after their final assessment.

\section{Participants}

Eligible patients were aged $\geq 18$ years, fluent in German, chemotherapy-naïve and newly diagnosed with gastrointestinal cancer (i.e. oesophagus, stomach, pancreas, gallbladder, bile duct, small and large intestines, rectum and anus, and cancer of unknown primary with metastases in the gastrointestinal tract). Exclusion criteria were impaired capability of self-care (Eastern Co-Operative Oncology Group (ECOG) Score $\geq 3$ ), severe psychological disorder (schizophrenia, substance abuse, severe depression or severe anxiety disorder), acute medical condition, chronic skin or lung disease (or dyspnoea or rash before starting treatment) and treatment with epidermal growth factor receptor antibodies.

We conducted a stratified randomization with block sizes of 2 and 4 . Prior to first enrolment, a research assistant generated the allocation sequence using a computer program $\left({ }^{19}\right)$, and prepared sequentially numbered, opaque, sealed envelopes. The stratum, distress, was assessed using a 10-point distress thermometer at T1pre $\left(<5\right.$ low vs. $\geq 5$ high $\left.{ }^{20}\right)$. Unaware of block-sizes, the trained healthcare professional performed the 1:1 group allocation after T1pre assessment by opening the envelope in front of the patient.

The trained healthcare professional performed both randomization and intervention and was therefore not blinded. Patients were unaware of the specific research question and the content of the other intervention.

Except for manualized reminder calls for outstanding questionnaires in isolated cases, the study team did not interact with patients for the outcome assessments (data collection is further detailed in the study protocol ${ }^{17}$ ).

As indicated in the study protocol ${ }^{17}$, a sample of $\mathrm{n}=90$ was required to detect a between-group effect of medium size (Cohen's $\left.d=0.6\right)$, given $80 \%$ power and $5 \%$ alpha-error (two-tailed). Considering a potential drop-out rate of $10 \%$, we aimed at including $\mathrm{N}=100 \mathrm{patients.}$

\section{Outcomes}

Our primary outcome was group difference in AEs at 10 days (T2) and 12 weeks (T3) after onset of chemotherapy, assessed with the Generic Assessment of Side Effects (GASE), which demonstrated high internal consistency and validity ${ }^{21}$. Patients rated the severity of seven symptoms in the past 7 days from 0 not present to 10 severe. Four symptoms, mainly fluoropyrimidines and/or platinum agents, used to treat gastrointestinal tumours (nausea, vomiting, diarrhoea, and fatigue) were specific to the most common chemotherapeutics ${ }^{22-26}$. Three 
symptoms (headache, shortness-of-breath and rash) were non-specific to chemotherapy ${ }^{22-26}$. The item range was increased (original GASE: $0-3)$ in the interest of higher outcome sensitivity.

Secondary outcomes included perceived control of $A E s^{17}$, misattribution tendency ${ }^{17}$, use of co-medication to treat $A E s$ (yes/no), and clinician-rated toxicity (Common Terminology Criteria for Adverse Events (CTCAE) Version $4.03{ }^{27}$ ) at T2 and T3. Compliance intention and attitude towards chemotherapy were assessed at T2.

\section{Further assessments}

Patients' expectations of the severity (AE expectations) and expected control of each AE (control expectations) were assessed at T1pre and T1post. Items were rated on a scale of 0 not at all to 10 completely. Cronbach's alpha indicated good internal consistency (AE expectations: a $=.89-.91$; Control expectations: $\mathrm{a}=.88-.91)$.

Sociodemographic data, distress level, tumour site and chronic somatic diseases and desire for information were self-reported at T1Pre. Tumour stage (UICC ${ }^{28}$ ), treatment aim and chemotherapy regime at T1pre and tumour progression (yes/no) at T2 and T3 were retrieved from medical records. At T1post, patients indicated their satisfaction with the preceding conversation. As a manipulation check, the nocebo group was asked to give free-text descriptions of the nocebo effect at T1post and T2.

\section{Statistical analysis}

All analyses were conducted with the intention-to-treat sample. Among completed questionnaires, missing values ranged between $1 \%$ and $6.3 \%$ per item, and were imputed through multiple imputation using the fully conditional specification method. Both death and discontinuation of chemotherapy were included as indicators ${ }^{29}$. We generated 15 imputed datasets and pooled parameters according to Rubin's rule ${ }^{30}$. Adjusted degrees of freedom were computed by hand and in alignment with the R package mice's procedures ${ }^{31}$. Comedication to treat AE (yes/no) was imputed from medical records. CTCAE data, missing for 27 and 40 patients at T2 and T3, were not imputed. SPSS version $25.0^{32}$ was used for data analyses and imputation.

We computed linear mixed models for repeated measures using restricted maximum-likelihood estimation and a variance component matrix type for our primary outcome AEs and our secondary outcomes control of AEs and misattribution tendency. The assessment timepoints T2 and T3 (level-1) were nested within patients (level-2). Fixed effects included Group, Time, Group x Time, Distress, and Cancer Staging. The intercept was included as a random effect. Group differences were examined via pairwise comparisons. Assumptions of linear models were checked prior analyses ${ }^{33}$.

We conducted regression analyses to examine the group difference in attitude towards chemotherapy (linear regression), compliance intention (Poisson regression), co-medication to treat AEs (yes/no; logistic regression). In all multivariate analyses, distress, cancer staging, and (if existent) the baseline of the respective outcome variable, were included as covariates ${ }^{17}$.

We calculated risk ratios for experiencing at least one AE based on the CTCAE.

To assess whether changes in expectations before and after the intervention were the underlying mechanisms of reduced AEs, we conducted mediation analyses. Two models were calculated: (1) group as predictor ( $\mathrm{X}$ ), AEs at T2 and T3, as outcomes ( $\mathrm{Y}$ ) and change in AE expectations (model 1 ) or change in control expectations (model 2 ) as the mediator. We obtained path a via regression analyses, and paths $b$ and c' via linear mixed models ${ }^{34}$. All effects were standardized. Mediation effects were examined using Monte Carlo simulations with 20,000 repetitions ${ }^{35,36}$; mediation was established if the confidence interval around the indirect effect did not contain zero.

Lastly, desire for information about AEs was examined as a moderator of the primary outcome by including the desire for information and desire for information $x$ group as additional fixed effects in the linear mixed models.

Sensitivity analyses of the primary outcome can be found in Supplementary Material A.

\section{Results}

\section{Patient flow}

Of all patients pre-screened by their oncologist, $\mathrm{n}=124$ were referred for eligibility assessment. Thereof, $N=100$ participants were randomized into the nocebo education $(E G ; n=49)$ and attention control $(C G ; n=51)$ groups. Participants received the respective intervention during the first course of chemotherapy, except for three who received it prior. The dropout rate was $30 \%$; by T3, $n=12(24.4 \%)$ and $n=18$ (35.3\%) patients in the EG and CG were lost. The range of completion was $4-98$ days after onset of chemotherapy $(M=22.1, S D=18.7)$ for 
T2 (scheduled: 10 days) and $73-225$ days $(M=111.1, S D=31.9)$ for T3 (scheduled: 12 weeks i.e. 84 days). Patient characteristics are detailed in Table 1.

\section{Table 1.}

Note. Sample characteristics at baseline. AEs = adverse events; CG = attention control group; EG = nocebo education group; UICC = Union for International Cancer Control. ${ }^{a}$ As overall sample size is $N=100$, percentages equal numbers. ${ }^{b}$ The healthcare professional delivered the nocebo education or conducted the quality of life interview in the attention control group. 'FOLFOX (leucovorin, 5-fluorouracil \& oxaliplatin), FUFOX (high dosage 5-fluorouracil, folic acid \& oxaliplatin), CAPOX (capecitabine + oxaliplatin), FLO (5-fluorouracil, leucovorin, oxaliplatin);

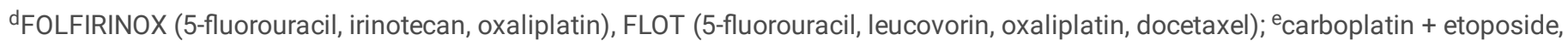

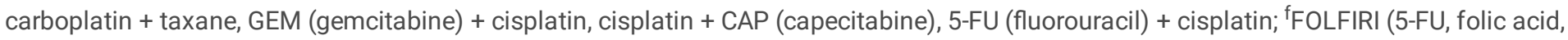

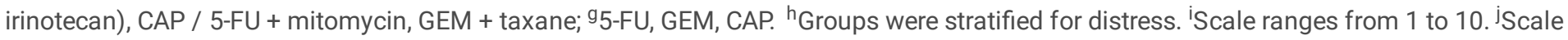
ranges from 0 to 10 , higher values indicate a more positive attitude. ${ }^{\text {S }}$ cale ranges from 0 to 10 , higher values indicate believing in the efficacy. 'Indicated for $\mathrm{n}=99$ patients at T1post.

\section{Primary outcome: adverse events}

At T2 and T3, four and one patients indicated no AEs (Figure 2). Among patients who did, the mean score was 14.83 (range: 1 - 36 ) at T2 and 17.61 (range: 3 - 44) at T3. Twenty-two and 12 patients reported no non-specific AEs at T2 and T3. The global scale showed a moderately positive correlation with total AEs at T2 $(r=0.62, p<.001)$ and T3 $(r=0.56, p<.001)$.

At T3, AEs in the EG were significantly lower, by 4.04 points (SE = 1.69), than in the CG (Table 2). Similarly, square root transformed nonspecific AEs were significantly lower in the EG at T3 by 0.39 points $(S E=0.18)$. Group differences in trend were found for both square-root transformed specific AEs the global scale at T3. At T2, AEs did not differ between groups.

\section{Table 2}

Group differences in adverse events, control of adverse events, and misattribution tendency 


\begin{tabular}{|c|c|c|c|c|c|}
\hline & \multirow{2}{*}{$\begin{array}{l}\text { Total Sample } \\
(N=100) \\
N^{a}\end{array}$} & \multicolumn{2}{|c|}{ EG $(n=49)$} & \multicolumn{2}{|c|}{$\mathrm{CG}(n=51)$} \\
\hline & & $N$ & $\%$ & $N$ & $\%$ \\
\hline Age, years $(M, S D)$ & $60.22(11.45)$ & 58.53 & 12.39 & 61.84 & 10.20 \\
\hline Gender (female) & 35 & 15 & 30.6 & 20 & 39.2 \\
\hline \multicolumn{6}{|l|}{ Education } \\
\hline$\leq 10$ year of school & 56 & 25 & 51 & 31 & 60.8 \\
\hline 13 years of school & 23 & 12 & 24.5 & 11 & 21.6 \\
\hline University degree & 21 & 12 & 24.5 & 9 & 17.6 \\
\hline \multicolumn{6}{|l|}{ Employment status } \\
\hline Employed & 51 & 27 & 55.1 & 24 & 47.1 \\
\hline Freelancer & 20 & 8 & 16.3 & 12 & 23.5 \\
\hline Homemaker & 5 & 2 & 4.1 & 3 & 5.9 \\
\hline Unemployed & 2 & 2 & 4.1 & 0 & 0 \\
\hline Pensioner & 22 & 10 & 20.4 & 12 & 23.5 \\
\hline \multicolumn{6}{|l|}{ Location } \\
\hline University Clinic Hamburg-Eppendorf & 91 & 45 & 91.8 & 46 & 90.2 \\
\hline Cooperating practice & 9 & 4 & 8.2 & 5 & 9.8 \\
\hline \multicolumn{6}{|l|}{ Healthcare professional ${ }^{b}$} \\
\hline BSc Psychologist (female) & 35 & 19 & 37.3 & 16 & 45.7 \\
\hline Medical doctoral candidate (male) & 65 & 32 & 62.7 & 33 & 32.7 \\
\hline \multicolumn{6}{|l|}{ Cancer Staging (UICC) } \\
\hline I & 4 & 2 & 4.1 & 2 & 3.9 \\
\hline II & 5 & 3 & 6.1 & 2 & 3.9 \\
\hline III & 37 & 14 & 28.6 & 23 & 45.1 \\
\hline IV & 54 & 30 & 61.2 & 24 & 47.1 \\
\hline \multicolumn{6}{|l|}{ Type of cancer } \\
\hline Upper gastrointestinal tract & 31 & 10 & 20.4 & 21 & 41.2 \\
\hline Lower gastrointestinal tract & 35 & 22 & 44.9 & 13 & 25.5 \\
\hline Gallbladder \& biliary tract & 8 & 4 & 8.2 & 4 & 7.8 \\
\hline Cancer of unknown primary & 3 & 2 & 4.1 & 1 & 2.0 \\
\hline Liver & 1 & 1 & 2.0 & 1 & 2.0 \\
\hline Pancreas & 10 & 10 & 20.4 & 11 & 21.6 \\
\hline \multicolumn{6}{|l|}{ Type of chemotherapy } \\
\hline Adjuvant & 25 & 14 & 28.6 & 11 & 21.6 \\
\hline Neoadjuvant & 24 & 9 & 18.4 & 15 & 29.4 \\
\hline Palliative & 49 & 24 & 49.0 & 25 & 49.0 \\
\hline Additive & 2 & 2 & 4.1 & 0 & 0 \\
\hline Additional radiation therapy & 12 & 5 & 10.2 & 7 & 13.7 \\
\hline
\end{tabular}




\begin{tabular}{|c|c|c|c|c|c|}
\hline & \multirow{2}{*}{$\begin{array}{l}\text { Total Sample } \\
(N=100) \\
N^{a}\end{array}$} & \multicolumn{2}{|c|}{ EG $(n=49)$} & \multicolumn{2}{|c|}{$\mathrm{CG}(n=51)$} \\
\hline & & $N$ & $\%$ & $N$ & $\%$ \\
\hline \multicolumn{6}{|l|}{ First-line chemotherapy regimen } \\
\hline Fluoropyrimidine/ platin doublet ${ }^{c}$ & 37 & 18 & 36.7 & 19 & 37.2 \\
\hline Fluoropyrimidine/ platin triplet ${ }^{d}$ & 14 & 5 & 10.2 & 9 & 17.7 \\
\hline Platin-based doublete & 25 & 16 & 32.7 & 9 & 17.7 \\
\hline & 7 & 2 & 4.1 & 5 & 9.8 \\
\hline Other doublets ${ }^{\dagger}$ & 11 & 7 & 14.3 & 4 & 7.8 \\
\hline Monotherapy ${ }^{g}$ & 6 & 1 & 2.0 & 5 & 9.8 \\
\hline \multicolumn{6}{|l|}{ Missing information } \\
\hline Physical comorbidity present & 40 & 17 & 34.7 & 23 & 45.1 \\
\hline High distress $(\geq 5)^{\mathrm{h}}$ & 71 & 35 & 71.4 & 36 & 70.6 \\
\hline Distress $(M, S D)^{\mathrm{i}}$ & $5.74(2.89)$ & 5.75 & 2.83 & 5.73 & 2.98 \\
\hline Compliance intention & $9.09(1.41)$ & 9.01 & 1.54 & 9.17 & 1.29 \\
\hline Attitude towards chemotherapy $(M, S D)^{\mathrm{j}}$ & $7.85(2.19)$ & 7.78 & 2.32 & 7.92 & 2.07 \\
\hline Perceived efficacy of the chemotherapy $(M, S D)^{\mathrm{k}}$ & $8.72(1.52)$ & 8.67 & 1.62 & 8.78 & 1.44 \\
\hline Desire for information about $\operatorname{AEs}(M, S D)^{1}$ & $6.92(3.01)$ & 6.98 & 2.93 & 6.86 & 3.11 \\
\hline Expected $\mathrm{AEs}(M, S D)^{\prime}$ & $3.85(1.86)$ & 3.94 & 1.73 & 3.75 & 2.00 \\
\hline Expected control of $A E s(M, S D)^{\prime}$ & $4.72(1.95)$ & 4.86 & 1.94 & 4.59 & 1.97 \\
\hline
\end{tabular}




\begin{tabular}{|c|c|c|c|c|c|c|c|c|c|c|c|c|c|c|}
\hline & \multicolumn{7}{|l|}{ T2 } & \multicolumn{7}{|l|}{ T3 } \\
\hline & \multicolumn{3}{|l|}{ CG } & \multicolumn{3}{|l|}{ EG } & \multirow{2}{*}{$\begin{array}{l}\text { Group } \\
\text { Comparison }\end{array}$} & \multicolumn{3}{|l|}{ CG } & \multicolumn{3}{|l|}{ EG } & \multirow{2}{*}{$\begin{array}{l}\text { Group } \\
\text { Comparison }\end{array}$} \\
\hline & $M$ & $S E$ & $N$ & $M$ & $S E$ & $N$ & & $M$ & $S E$ & $\mathrm{~N}$ & $M$ & $S E$ & $\mathrm{~N}$ & \\
\hline Total AEs & 14.87 & 1.19 & 51 & 13.58 & 1.20 & 49 & $\begin{array}{l}\text { Mean } \\
\text { difference: } \\
1.30,95 \% \mathrm{Cl} \\
{[-2.00,4.59]} \\
\text { Wald }= \\
0.77, d f= \\
87, p=44, \\
d=0.15\end{array}$ & 19.41 & 1.19 & 51 & 15.37 & 1.21 & 49 & $\begin{array}{l}\text { Mean } \\
\text { difference: } \\
4.04,95 \% \mathrm{Cl} \\
{[0.72,7.36]} \\
\text { Wald }= \\
2.39, d f= \\
86, p=.02, \\
d=0.48\end{array}$ \\
\hline Specific AEs & 3.14 & 0.14 & & 3.10 & 0.14 & & \multirow{2}{*}{$\begin{array}{l}\text { Mean } \\
\text { difference: } \\
0.04,95 \% \mathrm{Cl} \\
{[-0.34,0.41]} \\
\text { Wald }= \\
0.19, d f= \\
88, p=.85 \\
d=0.04\end{array}$} & 3.62 & 0.14 & & 3.26 & 0.14 & & \multirow{2}{*}{$\begin{array}{l}\text { Mean } \\
\text { difference: } \\
0.36,95 \% \mathrm{Cl} \\
{[-0.02,0.74]} \\
\text { Wald }= \\
1.84, d f= \\
85, p=.07 \\
d=0.37\end{array}$} \\
\hline Original scale & 8.85 & & & 8.62 & & & & & \multicolumn{3}{|l|}{9.65} & \\
\hline $\begin{array}{l}\text { Non-Specific } \\
\text { AEs }\end{array}$ & 2.29 & 0.13 & & 2.05 & 0.13 & & \multirow{2}{*}{$\begin{array}{l}\text { Mean } \\
\text { difference: } \\
0.24,95 \% \mathrm{Cl} \\
{[-0.11,0.59]} \\
\text { Wald }= \\
1.35, d f= \\
82, p=.18 \\
d=0.27\end{array}$} & 2.61 & 0.12 & & 2.22 & 0.13 & & \multirow{2}{*}{$\begin{array}{l}\text { Mean } \\
\text { difference: } \\
0.39,95 \% \mathrm{Cl} \\
{[0.04,0.73]} \\
\text { Wald }= \\
2.19, d f= \\
83, p= \\
.03, d=0.44\end{array}$} \\
\hline Original scale & 4.26 & & & 3.22 & & & & \multicolumn{3}{|l|}{5.81} & \multicolumn{3}{|l|}{3.94} & \\
\hline $\begin{array}{l}\text { Global AE } \\
\text { scale }\end{array}$ & 4.43 & 0.37 & 51 & 3.90 & 0.37 & 49 & $\begin{array}{l}\text { Mean } \\
\text { difference: } \\
0.53,95 \% \mathrm{Cl} \\
{[-0.49,1.56]} \\
\text { Wald }= \\
1.02, d f= \\
86, p=0.31 \\
d=0.20\end{array}$ & 5.45 & 0.37 & 51 & 4.32 & 0.39 & 49 & $\begin{array}{l}\text { Mean } \\
\text { difference: } \\
1.01,95 \% \mathrm{Cl} \\
{[-0.02,2.05]} \\
\text { Wald }= \\
1.92, d f= \\
84, p=.06 \\
d=0.38\end{array}$ \\
\hline $\begin{array}{l}\text { Control of } \\
\text { AEs }\end{array}$ & 1.88 & 0.07 & 48 & 1.93 & 0.07 & 48 & \multirow{2}{*}{$\begin{array}{l}\text { Mean } \\
\text { difference: } \\
-0.05,95 \% \\
\text { Cl }[-0.23 \\
0.14], \text { Wald } \\
=0.51, d f= \\
83, p=.61 \\
d=0.10\end{array}$} & 1.76 & 0.06 & 51 & 1.77 & 0.07 & 48 & \multirow{2}{*}{$\begin{array}{l}\text { Mean } \\
\text { difference: } \\
-0.01,95 \% \\
\text { CI [-0.19, } \\
0.17] \text {, Wald } \\
=0.07, d f= \\
87, p=.95, d \\
=0.01\end{array}$} \\
\hline Original scale & 2.53 & & & 2.71 & & & & 2.11 & & & 2.13 & & & \\
\hline $\begin{array}{l}\text { Misattribution } \\
\text { tendency }\end{array}$ & 2.08 & 0.08 & 42 & 1.89 & 0.08 & 36 & $\begin{array}{l}\text { Mean } \\
\text { difference: } \\
0.19,95 \% \mathrm{Cl} \\
{[-0.04,0.42]} \\
\text { Wald }=\end{array}$ & 2.21 & 0.07 & 47 & 2.08 & 0.08 & 41 & $\begin{array}{l}\text { Mean } \\
\text { difference: } \\
0.13,95 \% \mathrm{Cl} \\
{[-0.07,0.34] \text {, }} \\
\text { Wald }=\end{array}$ \\
\hline Original scale & 3.31 & & & 2.55 & & & $\begin{array}{l}1.65, d f= \\
58, p=.10 \\
d=0.38\end{array}$ & 3.89 & & & 3.33 & & & $\begin{array}{l}1.27, d f= \\
72, p=.21 \\
d=0.27\end{array}$ \\
\hline
\end{tabular}

Note. Pooled means and standard errors of linear mixed models after adjusting for distress and cancer staging. The primary outcome, adverse events (AEs), was computed as a sum-score of 7 symptoms x 10-point severity-scale resulting in a scale range of 0 to 70 . Accordingly, its (non-transformed) subscales specific AEs and non-specific AEs have ranges of 0-40 and 0-30, respectively. The global scale ranged from 0 to 10 . Control of adverse events ranged from 0 to 10 and was computed for patients who reported at least one adverse event. Misattribution tendency ranged from 0 to 10 and was computed for patients who reported non-specific adverse effects. Means on the original scales were obtained by back transforming the estimates. Significant differences were indicated in bold. Primary outcomes are shaded. 
$\mathrm{AE}=$ adverse event; $\mathrm{T} 2$ = 10 days after onset of chemotherapy; $\mathrm{T} 3$ = 12 weeks after onset of chemotherapy; $\mathrm{CG}=$ attention control group; $\mathrm{EG}=$ nocebo education group; $\mathrm{Cl}=$ confidence interval, $d$ = Cohen's $\mathrm{d}$.

\section{Secondary outcomes}

On average, patients rated their ability to control AEs and their misattribution tendency as low (Table 2). Linear mixed models indicated no significant group differences in perceived control of AEs and misattribution tendency (Table 2).

Patients' overall attitude towards their chemotherapy was positive $(M=7.45, S D=2.03)$ and compliance intention was very high $(M=8.76$, $S D=1.41$ ), with $45 \%$ of patients indicating a maximum score of 10 . We found no group difference for both variables at T2 (attitude towards chemotherapy: $\beta=0.06,95 \% \mathrm{Cl}[-0.34 ; 0.46], p=.79$; compliance intention: $O R=0.76,95 \% \mathrm{Cl}[0.51 ; 1.19], p=.24)$.

At T2 and T3, $n=52$ (of $n=82 ; 63.4 \%$ ) and $n=54$ (of $n=83,65.0 \%$ ) patients reported using co-medication to treat AEs, with no significant group differences (T2: $O R=1.98,95 \% \mathrm{Cl}[0.77,5.12], p=.16$, Nagelkerke's $R^{2}$ ModelT2 = .09; percentage of correctly predicted cases: $68.3 \%$; T3: $O R=0.73,95 \% \mathrm{Cl}[0.29,1.85], p=.51$, Nagelkerke's $R^{2}$ ModelT3 $=.06$; percentage of correctly predicted cases: $\left.66.3 \%\right)$.

Descriptive statistics of clinician-rated AEs according to CTCAE 27 are given in Table 3 . The risk ratio for developing at least one AE when allocated to EG vs. CG was 1.14 at T2 $(95 \% \mathrm{Cl}[0.73,1.78], p=.57)$, and 1.25 at T3 $(95 \% \mathrm{Cl}[0.72 ; 2.16], p=.43)$.

Table 3

\begin{tabular}{|c|c|c|c|c|c|c|c|c|c|c|c|c|c|c|}
\hline & \multicolumn{7}{|l|}{$\mathrm{T} 2$} & \multicolumn{7}{|l|}{ T3 } \\
\hline & \multicolumn{3}{|c|}{$E G(n=38)$} & \multicolumn{3}{|c|}{ CG $(n=35)$} & \multirow[t]{2}{*}{ Sum } & \multicolumn{3}{|c|}{$E G(n=31)$} & \multicolumn{3}{|c|}{ CG $(n=29)$} & \multirow[t]{2}{*}{ Sum } \\
\hline & G1 & $\mathrm{G} 2$ & G3 & G1 & $\mathrm{G} 2$ & G3 & & G1 & $\mathrm{G} 2$ & G3 & G1 & $\mathrm{G} 2$ & G3 & \\
\hline Nausea & 6 & 6 & 0 & 4 & 1 & 0 & 17 & 4 & 2 & 0 & 3 & 0 & 1 & 10 \\
\hline Vomiting & 0 & 2 & 0 & 3 & 0 & 0 & 19 & 0 & 0 & 0 & 1 & 0 & 1 & 2 \\
\hline Diarrhoea & 4 & 2 & 0 & 7 & 2 & 0 & 15 & 5 & 1 & 0 & 2 & 1 & 0 & 9 \\
\hline Fatigue & 4 & 6 & 0 & 1 & 1 & 0 & 12 & 4 & 5 & 0 & 3 & 1 & 1 & 14 \\
\hline Headache & 0 & 0 & 0 & 0 & 1 & 0 & 1 & 0 & 0 & 0 & 1 & 0 & 0 & 1 \\
\hline Shortness-of-breath & 0 & 1 & 0 & 0 & 0 & 0 & 1 & 2 & 0 & 0 & 1 & 0 & 0 & 3 \\
\hline \multirow[t]{2}{*}{ Rash } & 2 & 0 & 0 & 0 & 0 & 0 & 2 & 1 & 0 & 0 & 0 & 1 & 0 & 2 \\
\hline & \multicolumn{3}{|c|}{$\geq 1 \mathrm{AE}: \mathrm{n}=21$} & \multicolumn{4}{|c|}{$\geq 1 \mathrm{AE}: \mathrm{n}=17$} & \multicolumn{3}{|c|}{$\geq 1 \mathrm{AE}: \mathrm{n}=16$} & \multicolumn{4}{|c|}{$\geq 1 \mathrm{AE}: \mathrm{n}=12$} \\
\hline
\end{tabular}

Note. Clinician-rated adverse events of chemotherapy at T2 and T3 according to Common Terminology Criteria for Adverse Events. AE = adverse events; $C G$ = attention control group; $E G$ = nocebo education group; $T 2=10$ days after onset of chemotherapy; $\mathrm{T} 3=12$ weeks after onset of chemotherapy. G1 = Grade 1 "Mild; asymptomatic or mild symptoms; clinical or diagnostic observations only; intervention not indicated"; G2 = Grade 2 "Moderate; minimal, local or non-invasive intervention indicated; limiting age-appropriate instrumental activities of daily living; G3 = Grade 3 "Severe or medically significant but not immediately life-threatening; hospitalization or prolongation of hospitalization indicated; disabling; limiting self-care activities of daily living (defined by the National Institutes of Health, National Cancer Institute, 2009).

\section{Mechanisms of change}

We examined whether the effect of group on total AEs was mediated by a T1pre to T1post change in expected $A E s\left(M_{1}\right)$ and expected control of AEs $\left(\mathrm{M}_{2}\right)$. Expected AEs at T1 post correlated with total AEs at T2 $(r=0.27, p=.01)$ and T3 $(r=0.32, p=.002)$. Expected control correlated with total AEs in trend at T2 $(r=0.20, p=.06)$, and significantly at T3 $(r=0.26, p=.01)$. As the Monte Carlo test of mediation neither indicated an indirect effect of change in expected $A E s\left(\mathrm{M}_{1} ; 95 \% \mathrm{Cl}[-0.04 ; 0.04]\right)$, nor of change in expected control of $\mathrm{AEs}\left(\mathrm{M}_{2}\right)(95 \% \mathrm{Cl}[-0.20 ; 0.04])$. There was no effect of group $(X)$ on change in expected $A E s\left(M_{1}\right)\left(P a t h A_{1}: \beta=-0.09,95 \% \mathrm{Cl}[-0.49 ; 0.31], p=.65\right)$, or of change in expected AEs $\left(\mathrm{M}_{1}\right)$ on total AEs $(\mathrm{Y})$ (Path $\mathrm{B}_{1}$ (linear mixed model): standardized estimate $\left.=.01,95 \% \mathrm{Cl}[-0.15 ; 0.16], p=.95\right)$. ). The direct effect, i.e., 
when adjusting for the mediator and its baseline value, was significant (Path C': standardized estimate $=-0.47,95 \% \mathrm{Cl}[-0.84 ;-0.10], p=.01$ ), and comparable to the total effect $\mathrm{C}_{1}$ (standardized estimate $=-0.45,95 \% \mathrm{Cl}[-0.83 ;-0.08], p=.02$ ).

In contrast to $\mathrm{M}_{1}$, we found a significant effect of group $(X)$ on change in expected control $\left(\mathrm{M}_{2}\right)$ (Path $\mathrm{A}_{2}: \beta=0.62,95 \% \mathrm{Cl}[0.25,1.00], p=$ $.001)$. There was no effect of change in expected control $\left(\mathrm{M}_{2}\right)$ on total AEs $(\mathrm{Y})\left(\right.$ Path $\mathrm{B}_{2}$ : standardized estimate $=-0.11,95 \% \mathrm{Cl}[-0.28,0.07], p$ $=.23$ ). We found a significant total effect of group $(\mathrm{X})$ on total AEs ( $\mathrm{Y}$; standardized estimate $=-0.45,95 \% \mathrm{Cl}[-0.83 ;-0.08], p=.02)$. Compared to the total effect, we found a smaller direct effect of group $(X)$ on the outcome $(Y)$ after adjusting for $M_{2}$ (standardized estimate $=-0.40,95 \%$ Cl [-0.78; -0.01], $p=.046)$.

\section{Moderator of the intervention}

The interaction effect of Group $x$ Desire for information about AEs was not significant in the linear mixed model $(Y=$ total AEs), indicating no moderating effect of desire for information about AEs on total AEs (estimate: $0.06,95 \% \mathrm{Cl}[-0.43 ; 0.54], p=.82$ ).

\section{Evaluation of the intervention}

Patients in both groups rated the conversation as highly relevant (EG: $M=8.08, S D=1.90 ; C G: M=7.30, S D=2.19$; range: $0-10$ ) and indicated highly recommending it to other patients ( $E G: M=8.94, S D=1.44$; CG: $M=8.48, S D=1.87$; range: $0-10$ ).

\section{Discussion}

AEs of chemotherapy are susceptible to the nocebo effect (21). In the present study, we tested whether a nocebo education intervention could reduce $\mathrm{AEs}$ of chemotherapy in patients with $\mathrm{GI}$ cancer.

For our primary outcome, we found that 12 weeks (T3) after onset of chemotherapy, patients in the EG experienced significantly less AEs than the CG; specifically, they had less non-specific AEs and a trend towards less specific AEs. At 10-days (T2), there was no group difference. We found no group differences in control of AEs, misattribution of non-specific AEs to chemotherapy, attitude towards chemotherapy, compliance intention, use of co-medication to treat AEs, or risk of developing at least one AE. Further, information coping style did not influence the group difference in AEs.

\section{Adverse events}

This is the first study to show that the AEs of a medication can be reduced by educating patients about the nocebo effect, at a moderate effect size. Thereby, it confirms the clinical transferability of this concept from findings of two prior studies which experimentally induced symptoms that were subsequently reduced by nocebo education $(25,26)$.

Other trials aimed at reducing nocebo effects of verum medications have used positive framing methods. A recent review (51) shows that only one out of three studies which applied framing to AEs of a medication achieved a significant reduction of 2 out of 12 listed AEs (52). This underlines that medication AEs are difficult to modify, emphasizing the relative impact of our findings. Since our research is novel in its method of $A E$ modification as applied to verum medication, our results require replication.

In the context of psychosocial interventions for GI cancer patients, our intervention demonstrates favourable results. Mosher et al. (53) conducted a systematic review of 14 studies using interventions such as education, supportive care and relaxation in patients with colorectal cancer. Six of the eight interventions with QoL outcomes that included disease or treatment specific symptoms produced no effect (53). One study in which patients were provided with regular home visits for informational and emotional support compared to treatment as usual showed a reduction in fatigue $(p=0.048)$, but not other symptoms such as diarrhoea or shortness-of-breath (54). Likewise, training in progressive muscle relaxation improved overall colorectal cancer-related QoL $(p<0.001)$ and physical health specifically $(p<0.01)(55)$. Notably, both these interventions were considerably longer than ours yet their effects, unlike ours, were not controlled for attentiveness from the delivering healthcare professional. Findings from two further studies including a sample of primarily GI cancer (56) and hepatocellular-, gallbladder or cholangiocellular carcinoma (57) patients have the same trajectory: individually tailored psychotherapy sessions caused clinically relevant reductions in AEs (57) and a web-based collaborative care intervention with fortnightly follow-ups likewise produced small to medium effect sized improvements on pain and QoL (56). Therefore, we conclude that our intervention was effective and efficient compared to other psychosocial interventions. 
The prevalence of self-reported AEs resembles self-report from $n=142$ colorectal cancer patients receiving chemotherapy; the rank order of fatigue, diarrhoea, dyspnoea, rash and vomiting also mirror. (1). In a further study of self-report symptom prevalence in colorectal cancer patients, fatigue was also the most commonly, and rash and vomiting the most seldomly reported of the symptoms that we analysed (66). The high prevalence of fatigue reflects the lack of effective pharmacological treatment, whereas vomiting appears effectively controlled with pre-treatment antiemetics. The overall higher prevalence of symptoms at T3 than at T2 also corresponds to self-report findings by which chemotherapy AEs accumulate (1).

Our results demonstrate the clinical feasibility and the efficacy of the nocebo intervention in reducing AEs with clinically relevant, moderate to large effects sizes in comparison to a psychological control intervention. Further studies are needed to analyse potential action mechanisms of this treatment option.

\section{Secondary Outcomes}

Across groups, patients their ability to control AEs at 10-days and 12-weeks follow-up as low. Good strategies for symptom control lead to less AEs and better health-related QoL $(68,69)$, hence the large amount of cancer care interventions with this target [e.g., 27-29]. Improvement in perceived control has likewise been proposed as a pathway of AE reduction through nocebo education (73). Perhaps no group difference in control of AEs emerged in our study because the intervention did not target symptom control strategies.

Misattribution of non-specific AEs to chemotherapy was also low in both groups. One explanation would be to posit that all patients were well informed about which AEs to expect by their attending physicians, and therefore were not prone to misattribution. There are, however, several other aspects to consider. Prior findings on the impact of symptom misattribution focus on the immediate AEs after one-time, inert substance intake $(20,25)$, the psychological mechanism of which might be inapplicable to repeated chemotherapy. Further, misattribution is predicted by distress (20), therefore, our distress stratification may have decreased a potential intervention effect on misattribution. A positive attitude towards medicines, as displayed by our sample towards chemotherapy, has been shown to reduce misattribution; likewise, prior knowledge about specific chemotherapy AEs may have helped patients to identify non-specific AEs as such. To summarize, there are several potential explanations for the absence of symptom misattribution, preventing conclusions about the interplay of nocebo education and symptom misattribution in the current study.

We also analysed several clinically relevant outcomes. Compliance intention at 10-days was high across groups and, predictably, dependent upon its baseline level. We regarded compliance intention as a proxy for medication adherence. Our findings align with clinical adherence rates, with $78 \%$ of 3193 above 66-year-olds completing their chemotherapies for stage 3 colon carcinoma (75).

Group allocation was not predictive of whether or not patients reported using co-medication to treat AEs. The interpretability of this finding is limited by the large number of missing values on self-reports assessing co-medication use, which in turn were imputed from medical records. As these document the clinical oncologists' prescriptions, but not medications prescribed by general practitioners or obtained over-the-counter (e.g., loperamide for diarrhoea or dimenhydrinate for nausea), they only indirectly reflect the actual co-medication taken. Further, we did not assess dosage information so as to minimize the burden on patients, therefore possible dose reductions in response to lessened AEs could not be detected.

Unlike the self-reported total AEs, clinician-rated toxicity of AEs did not differ between groups. Disparity between self-and clinician-rated AEs of chemotherapy is well established $(58,59)$, and researchers argue that self-rated toxicity deserves clinical attention as it more closely reflects patients' QoL $(2,58)$.

\section{Further Assessments}

Desire for information about AEs did not moderate the intervention effect. Since a monitoring coping style (i.e., attending to and seeking information on symptoms) is associated with more AEs after admission of verum medications (29) as well as placebos (25), we assumed the intervention would buffer the influence of desire for information about AEs. Yet in line with recent findings (25), this relationship was not confirmed.

Patients rated both the intervention and attention control interview as highly relevant and indicated they would recommend it to other patients. Patients' free-text descriptions of the nocebo effect at 10-days, such as: "a self-fulfilling prophecy", verified that the majority gained a solid understanding. Given that the nocebo education information was only provided once and patients were under strain of their cancer symptoms, we consider this feedback positive.

\section{Strengths}

In designing this study, our decisions were led by pragmatism in order to maximize the clinical validity of our results (76). This strategy included recruitment through usual care, clinically determined in- and exclusion criteria, the liberality of which resulted in a heterogeneous and 
highly burdened sample with almost half of patients receiving palliative care, as well as an intervention that fit almost seamlessly into clinical routine. In effect, our findings have high external validity.

\section{Limitations}

We did not assess baseline levels of the seven analysed AEs, so it is unclear to which extent pre-existing symptoms influenced our results. For example, higher non-specific symptom burden at baseline can exacerbate nocebogenic AEs after starting a medication (20, 25), and symptoms of the underlying malignancy can mirror those of chemotherapy $(77,78)$, therefore the former could have been misattributed to the latter (79), reducing the observed intervention effect. The same effect may have occurred within the study period in patients who received concurrent radiation therapy, in that radiation sunburn may have been misinterpreted as rash due to chemotherapy.

We did not control for the AE information patients received from their attending physician during IC, which can vary considerably (80) and have a substantial effect on patients' experience of AEs (73). Finally, several items used were self-developed and not subjected to prior psychometric evaluation, limiting their comparability.

\section{Conclusions}

In severely ill, burdened patients, we showed that a single education session about the nocebo effect reduced the AEs of chemotherapy. In addition to specific AEs, many patients in our sample suffered from pharmacologically unlikely non-specific AEs, emphasizing that the latter must not be underestimated in their potential negative impact to patients' health. While our results are promising, they require replication to expand our current knowledge of modifying nocebo responses to medications by means of psychoeducation and cognitive reappraisal. Our intervention was integrated seamlessly into clinical routine and has the conceptual flexibility to be applied in various clinical settings. Nocebo education by no means replaces treatment such as co-medication for severe AEs; it rather serves as a low-level, patient empowering, supplementary measure. The potential of this line of research is that knowledge of the nocebo effect and coping with negative expectations becomes inherent to what we consider an informed patient.

\section{Abbreviations}

\begin{tabular}{ll} 
AEs & adverse events \\
\hline CG & attention control group \\
\hline CI & confidence interval \\
\hline CTCAE & Common Terminology Criteria for Adverse Events \\
\hline ECOG & Eastern Co-Operative Oncology Group \\
\hline EG & nocebo education group \\
\hline FACT-G & Functional Assessment of Cancer Therapy general \\
\hline GASE & Generic Assessment of Side Effects \\
\hline GI & gastrointestinal \\
\hline PP & per-protocol \\
\hline QoL & quality of life \\
\hline UICC & Union for International Cancer Control
\end{tabular}

\section{Declarations}

\section{Ethics approval and consent to participate:}

The trial was approved by the University of Hamburg ethics committee (ID: 2015_03). All methods were carried out in accordance with the Declaration of Helsinki. All participants provided written informed consent prior enrolment.

\section{Consent for publication:}

Not applicable 


\section{Availability of data and materials:}

The datasets used and/or analysed during the current study are available from the corresponding author on reasonable request.

\section{Competing interests:}

The authors declare that they have no competing interests.

\section{Funding:}

This work was supported by a research grant to YN from the Foundation for the Science of the Therapeutic Encounter (F-STE), [grant number: 1705-101]. The funder had no role in the design of the study, the collection, analysis, interpretation of data, and manuscript writing.

\section{Authors' contributions:}

Conceptualization: YN

Methodology: YN, YP

Investigation: $\mathrm{AH}, \mathrm{TM}$

Visualization: YP, TM

Funding acquisition: YN

Project administration: YN

Supervision: YN

Writing - original draft: TM

Writing - review \& editing: YP, YN, AH, AS, KO

All authors read and approved the final manuscript.

\section{Acknowledgements:}

We would like to thank Ted Kaptchuk who provided us with valuable guidance throughout the course of this project. We would also like to thank Melanie Salm for her support in data acquisition and the Oncology Outpatient Clinic of the University Medical Centre HamburgEppendorf for accommodating our research.

\section{References}

1. Pearce A, Haas M, Viney R et al. Incidence and severity of self-reported chemotherapy side effects in routine care: A prospective cohort study. PLoS One 2017; 12(10):e0184360.

2. Carlotto A, Hogsett VL, Maiorini EM et al. The Economic Burden of Toxicities Associated with Cancer Treatment: Review of the Literature and Analysis of Nausea and Vomiting, Diarrhoea, Oral Mucositis and Fatigue. Pharmacoeconomics 2013; 31(9):753-766.

3. Wagland R, Richardson A, Ewings S et al. Prevalence of cancer chemotherapy-related problems, their relation to health-related quality of life and associated supportive care: a cross-sectional survey. Support. Care Cancer 2016; 24(12):4901-4911.

4. Lorusso D, Bria E, Costantini A et al. Patients' perception of chemotherapy side effects: Expectations, doctor-patient communication and impact on quality of life - An Italian survey. Eur. J. Cancer Care (Engl). 2017; 26(2):e12618.

5. Jacobs JM, Ream ME, Pensak N et al. Patient Experiences With Oral Chemotherapy: Adherence, Symptoms, and Quality of Life. J Natl Compr Canc Netw 2019; 17(3):221-228.

6. Clarke G, Johnston S, Corrie P et al. Withdrawal of anticancer therapy in advanced disease: a systematic literature review. BMC Cancer $2015 ; 15: 892$. 
7. Mahr A, Golmard C, Pham E et al. Types, frequencies, and burden of nonspecific adverse events of drugs: analysis of randomized placebo-controlled clinical trials. Pharmacoepidemiol. Drug Saf. 2017; 26(7):731-741.

8. Barsky A, Saintfort R, Rogers M, Borus J. Nonspecific Medication Side Effects and the Nocebo Phenomenon. JAMA - J. Am. Med. Assoc. 2002; 10(1):133-134.

9. Chvetzoff G, Tannock IF. Placebo effects in oncology. J. Natl. Cancer Inst. 2003; 95(1):19-29.

10. L. C, D. F. Nocebo effects, patient-clinician communication, and therapeutic outcomes. JAMA - J. Am. Med. Assoc. 2012; 307(6):567568.

11. Nestoriuc $Y$, von Blanckenburg $P$, Schuricht $F$ et al. Is it best to expect the worst? Influence of patients' side-effect expectations on endocrine treatment outcome in a 2-year prospective clinical cohort study. Ann. Oncol. 2016; 27(10):1909-1915.

12. Shedden-Mora MC, Pan Y, Heisig SR et al. Optimizing expectations about endocrine treatment for breast cancer: Results of the randomized controlled psy-breast trial. Clin. Psychol. Eur. 2020; 2(1):1-20.

13. Pan Y, Kinitz T, Stapic M, Nestoriuc Y. Minimizing Drug Adverse Events by Informing About the Nocebo Effect-An Experimental Study. Front. Psychiatry 2019; 10(July):1-12.

14. Crichton F, Petrie KJ. Health complaints and wind turbines: The efficacy of explaining the nocebo response to reduce symptom reporting. Environ. Res. 2015; 140:449-455.

15. Miceli J, Geller D, Tsung A et al. Illness perceptions and perceived stress in patients with advanced gastrointestinal cancer. Psychooncology. 2019; 28(7):1513-1519.

16. van Gestel YRBM, Lemmens VEPP, de Hingh IHJT et al. Influence of Comorbidity and Age on 1-, 2-, and 3-Month Postoperative Mortality Rates in Gastrointestinal Cancer Patients. Ann. Surg. Oncol. 2013; 20(2):371-380.

17. Quidde J, Pan Y, Salm M et al. Preventing adverse events of chemotherapy by educating patients about the nocebo effect (RENNO study) - study protocol of a randomized controlled trial with gastrointestinal cancer patients. BMC Cancer 2018; 18(1):1-8.

18. Oken MM, Creech RH, Tormey DC et al. Toxicity and response criteria of the Eastern Cooperative Oncology Group. Am. J. Clin. Oncol. 1982; 5(6):649-656.

19. Abramson JH. WINPEPI (PEPI-for-Windows): computer programs for epidemiologists. Epidemiol. Perspect. Innov. 2004; 1(1):6.

20. Schneider S, Moyer A, Knapp-Oliver S et al. Pre-intervention distress moderates the efficacy of psychosocial treatment for cancer patients: A meta-analysis. J. Behav. Med. 2010; 33(1):1-14.

21. Rief W, Barsky AJ, Glombiewski JA et al. Assessing general side effects in clinical trials: reference data from the general population. Pharmacoepidemiol. Drug Saf. 2011; 20:405-415.

22. de Gramont A, Figer A, Seymour M et al. Leucovorin and fluorouracil with or without oxaliplatin as first-line treatment in advanced colorectal cancer. J. Clin. Oncol. 2000; 18(16):2938-2947.

23. Díaz-Rubio E, Tabernero J, Gómez-España A et al. Phase III study of capecitabine plus oxaliplatin compared with continuous-infusion fluorouracil plus oxaliplatin as first-line therapy in metastatic colorectal cancer: Final report of the Spanish Cooperative Group for the treatment of digestive tumors trial. J. Clin. Oncol. 2007; 25(27):4224-4230.

24. Cunningham D, Starling N, Rao S et al. Capecitabine and oxaliplatin for advanced esophagogastric cancer. N. Engl. J. Med. 2008; 358(1):36-46.

25. Conroy T, Desseigne F, Ychou M et al. FOLFIRINOX versus gemcitabine for metastatic pancreatic cancer. N. Engl. J. Med. 2011; 364(19):1817-1825.

26. Valle J, Wasan H, Palmer DH et al. Cisplatin plus gemcitabine versus gemcitabine for biliary tract cancer. N. Engl. J. Med. 2010; 362(14):1273-1281.

27. National Cancer Institute. Common Terminology Criteria for Adverse Events (CTCAE). Version 4.03., 2010.

28. TNM I UICC. [https://www.uicc.org/resources/tnm].

29. Biering K, Hjollund NH, Frydenberg M. Using multiple imputation to deal with missing data and attrition in longitudinal studies with repeated measures of patient-reported outcomes. Clin. Epidemiol. 2015; 7(January):91-106.

30. Rubin DB. Multiple imputation for nonresponse in surveys, John Wiley \& Sons, 2004.

31. Van Buuren S. Flexible imputation of missing data, CRC press, 2018.

32. IBM Corp. IBM SPSS Statistics for Windows. 2017.

33. Garson GD. Multilevel modeling: applications in STATA, IBM SPSS, SAS, R, \& HLM, Sage Publications, Inc, 2019.

34. Baron R, Kenny D. The moderator-mediator variable distinction in social psychological research: Conceptual, strategic, and statistical considerations. J. Pers. Soc. Psychol. 1986; 51:1173-1182.

Page 14/17 
35. Selig JP, Preacher KJ. Monte Carlo method for assessing mediation: An interactive tool for creating confidence intervals for indirect effects. 2008.

36. MacKinnon DP, Lockwood CM, Hoffman JM et al. A comparison of methods to test mediation and other intervening variable effects. Psychol. Methods 2002; 7(1):83.

37. Barnes K, Faasse K, Geers AL et al. Can Positive Framing Reduce Nocebo Side Effects? Current Evidence and Recommendation for Future Research. Front. Pharmacol. 2019. doi:10.3389/fphar.2019.00167.

38. O'Connor AM, Pennie RA, Dales RE. Framing effects on expectations, decisions, and side effects experienced: The case of influenza immunization. J. Clin. Epidemiol. 1996; 49(11):1271-1276.

39. Mosher CE, Winger JG, Given BA et al. A systematic review of psychosocial interventions for colorectal cancer patients. Support. Care Cancer 2017; 25(7):2349-2362.

40. Ross L, Thomsen BL, Karlsen R V. et al. A randomized psychosocial intervention study on the effect of home visits on the well-being of Danish colorectal cancer patients - The INCA project. Psychooncology. 2005; 14(11):949-961.

41. Cheung YL, Molassiotis A, Chang AM. The effect of progressive muscle relaxation training on anxiety and quality of life after stoma surgery in colorectal cancer patients. Psychooncology. 2003; 12(3):254-266.

42. Steel JL, Geller DA, Kim KH et al. Web-based collaborative care intervention to manage cancer-related symptoms in the palliative care setting. Cancer 2016; 122(8):1270-1282.

43. Steel JL, Nadeau K, Olek M, Carr BI. Preliminary results of an individually tailored psychosocial intervention for patients with advanced hepatobiliary carcinoma. J. Psychosoc. Oncol. 2007; 25(3):19-42.

44. Pettersson G, Berterö C, Unosson M, Börjeson S. Symptom prevalence, frequency, severity, and distress during chemotherapy for patients with colorectal cancer. Support. Care Cancer 2014; 22(5):1171-1179.

45. Burish TG, Tope DM. Psychological techniques for controlling the adverse side effects of cancer chemotherapy: Findings from a decade of research. J. Pain Symptom Manage. 1992. doi:10.1016/0885-3924(92)90062-M.

46. Scott DA, Mills M, Black A et al. Multidimensional rehabilitation programmes for adult cancer survivors. Cochrane Database Syst. Rev. 2013. doi:10.1002/14651858.CD007730.pub2.

47. Webster RK, Weinman J, Rubin GJ. Medicine-related beliefs predict attribution of symptoms to a sham medicine: A prospective study. Br. J. Health Psychol. 2018; 23(2):436-454.

48. Dobie SA, Baldwin L-M, Dominitz JA et al. Completion of Therapy by Medicare Patients With Stage III Colon Cancer. JNCI J. Natl. Cancer Inst. 2006; 98(9):610-619.

49. Fromme EK, Eilers KM, Mori M et al. How accurate is clinician reporting of chemotherapy adverse effects? A comparison with patientreported symptoms from the Quality-of-Life Questionnaire C30. J. Clin. Oncol. 2004; 22(17):3485-3490.

50. Greimel ER, Bjelic-Radisic V, Pfisterer J et al. Toxicity and quality of life outcomes in ovarian cancer patients participating in randomized controlled trials. Support. Care Cancer 2011; 19(9):1421-1427.

51. Miller SM. Monitoring versus blunting styles of coping with cancer influence the information patients want and need about their disease. Cancer 1980; 76(2):167-177.

52. Loudon K, Treweek S, Sullivan F et al. The PRECIS-2 tool: Designing trials that are fit for purpose. BMJ 2015. doi:10.1136/bmj.h2147.

53. Clinical presentation, diagnosis, and staging of colorectal cancer - UpToDate. [https://www.uptodate.com/contents/clinical-presentationdiagnosis-and-staging-of-colorectal-cancer?search=colorectal cancer\&source=search_result\&selectedTitle=1 150\&usage_type=default\&display_rank=1].

54. Clinical features, diagnosis, and staging of gastric cancer - UpToDate. [https://www.uptodate.com/contents/clinical-features-diagnosisand-staging-of-gastric-cancer?search=gastric

cancer\&source=search_result\&selectedTitle=1 150\&usage_type=default\&display_rank=1\#H45647872].

55. Fine S, Johnston C. Drug and placebo side effects in methylphenidate-placebo trial for attention deficit hyperactivity disorder. Child Psychiatry Hum. Dev. 1993; 24(1):25-30.

56. Rodriguez KL, Gambino FJ, Butow PN et al. 'It's going to shorten your life': framing of oncologist-patient communication about prognosis. Psychooncology. 2008; 17(3):219-225.

57. Barsky AJ. The iatrogenic potential of the physician's words. JAMA - J. Am. Med. Assoc. 2017; 318(24):2425-2426.

\section{Figures}




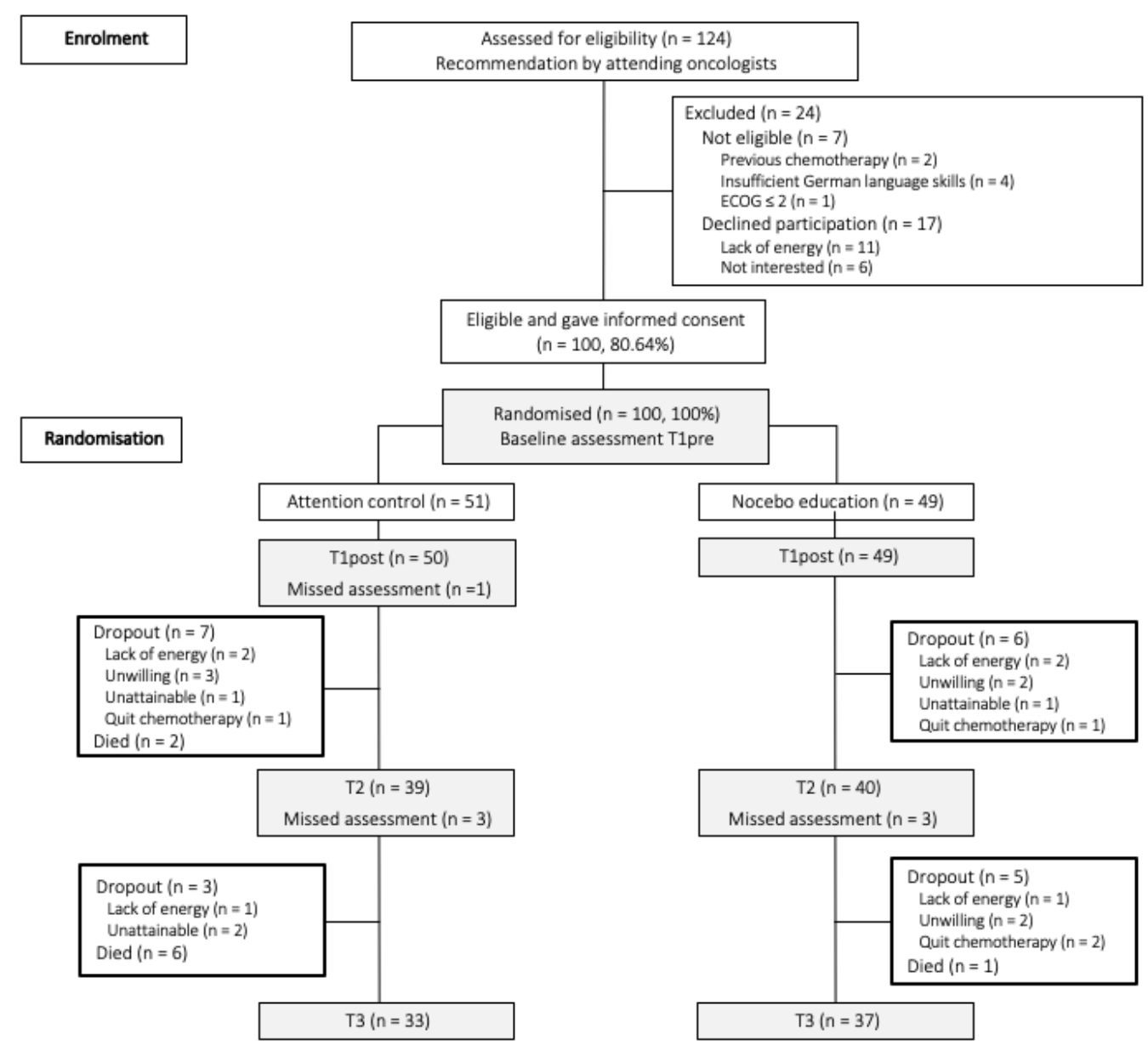

Analysed

Attention control $(n=51)$

Nocebo education $(n=49)$

\section{Figure 1}

CONSORT diagram. ECOG = Eastern Co-operative Oncology Group; values $\leq 2$ indicate limited capability of self-care. Assessment points are shaded. T1pre and T1post = immediately pre- and post-intervention, $\mathrm{T} 2=10$ days after onset of chemotherapy and $\mathrm{T} 3=12$ weeks after onset of chemotherapy. Missed assessment $=$ patients who missed the respective assessment but remained enrolled. One patient in the attention control group discontinued chemotherapy but completed T3.
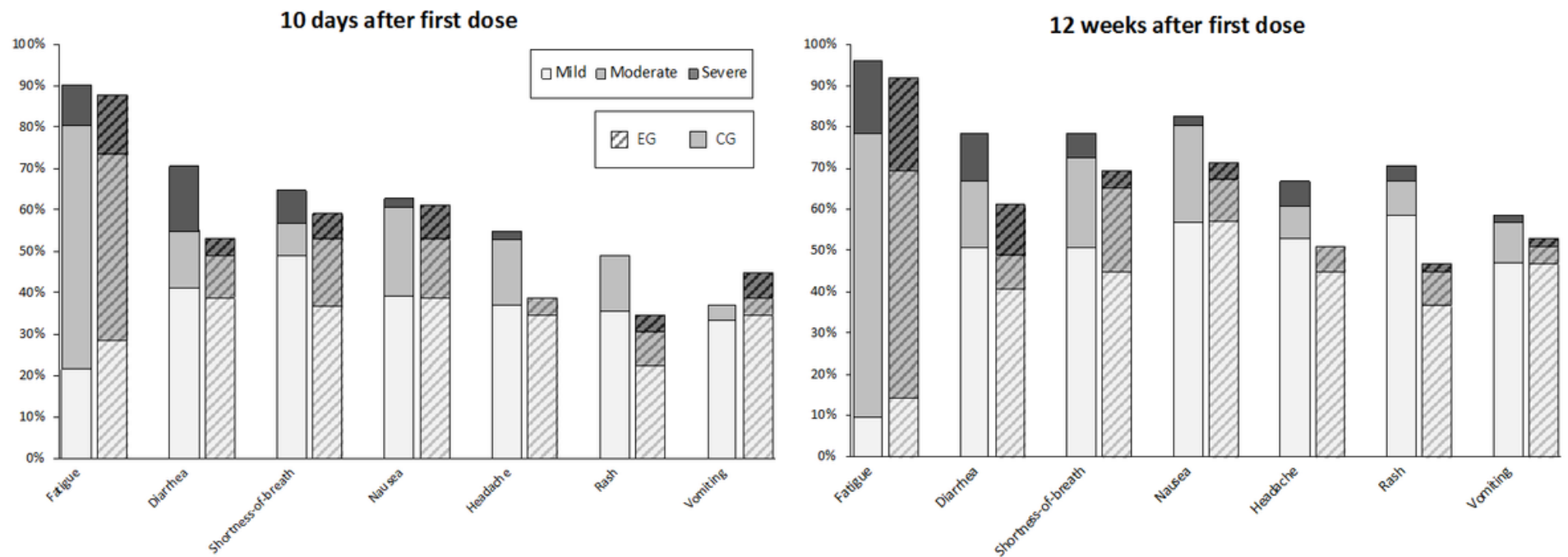

Page 16/17 
Figure 2

Severity and frequency of AEs at T2 and T3 (imputed data). Symptom severity was graded into $1-3$ mild, $4-7$ moderate and 8 - 10 severe. Specific adverse events: fatigue, diarrhoea, nausea and vomiting; non-specific adverse events: shortness-of-breath, headache and rash. T2 = 10 days after onset of chemotherapy; T3 = 12 weeks after onset of chemotherapy; $C G=$ attention control group ( $n=51$ ); EG = nocebo education group $(n=49)$.

\section{Supplementary Files}

This is a list of supplementary files associated with this preprint. Click to download.

- SupplementaryMaterialA.docx 